\title{
Avaliação dos efeitos da posição canguru nos sinais vitais em recém-nascidos pré-
}

\section{termo}

\author{
Assessment of the effects of Kangaroo Position on vital signs of pre term neonates
}

Evaluación de los efectos de la posición canguro sobre los signos vitales en recién nacidos

prematuros

Recebido: 05/01/2022 | Revisado: 14/01/2022 | Aceito: 17/01/2022 | Publicado: 19/01/2022

Elio Vieira de Oliveira

ORCID: https://orcid.org/0000-0001-6137-2527 Faculdades Integradas Santa Cruz de Curitiba, Brasil E-mail: vieiradeoleiveiraelio@gmail.com Pedro Leite de Melo Filho

ORCID: https://orcid.org/0000-0002-0102-5619 Centro Universitário Uniesp, Brasil E-mail: pedromelofilho56@gmail.com

Beatriz Essenfelder Borges

ORCID: https://orcid.org/0000-0001-9750-5635 Faculdades Integradas Santa Cruz de Curitiba, Brasil E-mail: biaessenfelder@gmail.com

\begin{abstract}
Resumo
Considera-se prematuro o recém-nascido com idade gestacional inferior a 37 semanas, sendo estes predispostos a morbidades e mortalidade. A Posição Canguru (PC) objetiva-se a minimizar os efeitos da condição de prematuridade. Avaliar os efeitos da PC sobre os sinais vitais em recém-nascidos pré-termo (RNPT). Foram avaliados 12 RNPT, estáveis, sem complicações neurológicas, cardíacas e/ou respiratórias, com idade gestacional entre 29 e 37 semanas, com peso entre 1.000 e 2.000 gramas. Após atingir o estado calmo segundo a escala Prechtl, os bebês foram avaliados e colocados na PC durante 30 minutos e em seguida reavaliados. As variáveis analisadas foram saturação periférica de oxigênio (SpO2), frequência respiratória (FR) e cardíaca $(\mathrm{FC})$, temperatura $(\mathrm{T})$ e pressão arterial $(\mathrm{PA})$. Observou-se diferenças significativas na SpO2 $(p=0,01), F R(p=0,008), F C(p=0,04)$ e $T(p=0,03)$. Já a PA, tanto a sistólica $(\mathrm{p}=0,47)$ quanto a diastólica $(\mathrm{p}=0,46)$ não apresentaram diferenças significativas. A PC proporcionou um aumento significativo da SpO2 e diminuição significativa da FR, FC e T nos RNPT avaliados.
\end{abstract}

Palavras-chave: Prematuridade; Recém-nascido; Posição canguru; Sinais vitais.

\begin{abstract}
Introduction: It is considered pre term the newborn under 37 weeks of gestational age, and thus more vulnerable to morbidity and mortality. The Kangaroo Position (KP) aims to minimize the effects of prematurity. To assess the KP effects on vital signs at pre and post applications of such method on hospitalized pre term neonates (PTN) of a large hospital in the city of Curitiba-PR. 12 PTN were assessed when stable, with no neurological, cardiologic, and/or respiratory complications, whit gestational ages between 29 to 37 weeks, weighing from $1000 \mathrm{~g}$ to $2000 \mathrm{~g}$. After reaching the calm and relaxed state, according to the Prechtl scale, the neonates were assessed and put on KP for 30 minutes and reassessed right after. The variables analyzed were peripheral oxygen saturation $(\mathrm{SpO} 2)$, heart rate, respiratory rate, body temperature, and blood pressure. Significant changes were observed in $\mathrm{SpO} 2(\mathrm{p}=0,01)$, respiratory rate $(\mathrm{p}=0,008)$, heart rate $(\mathrm{p} 0,04)$, and temperature $(\mathrm{p}=0,03)$. On the contrary, the blood pressure systolic $(\mathrm{p}=0,47)$, and diastolic $(\mathrm{p}=0,46)$ did not present significant changes. CONCLUSIONS: The KP caused an important increase in $\mathrm{SpO} 2$ and a significant decrease in respiratory rate, $\mathrm{HR}$, and temperature in the assessed PTN.
\end{abstract}

Keywords: Prematurity; Newborn; Kangaroo position; Vital signs.

\section{Resumen}

Un recién nacido con una edad gestacional inferior a 37 semanas se considera prematuro y estos están predispuestos a la morbilidad y la mortalidad. La Posición Canguro (PC) tiene como objetivo minimizar los efectos de la condición de prematuridad. Evaluar los efectos de la PC sobre los signos vitales en los recién nacidos prematuros (PTNB). Se evaluaron 12 lactantes prematuros estables, sin complicaciones neurológicas, cardíacas y / o respiratorias, con edad gestacional entre 29 y 37 semanas, con un peso entre 1000 y 2000 gramos. Después de alcanzar el estado de calma según la escala de Prechtl, los bebés fueron evaluados y colocados en el CP durante 30 minutos y luego reevaluados. Las variables analizadas fueron saturación periférica de oxígeno $(\mathrm{SpO} 2)$, frecuencia respiratoria $(\mathrm{RR})$ y frecuencia 
cardíaca (FC), temperatura (T) y presión arterial (PA). Se observaron diferencias significativas en $\mathrm{SpO} 2(\mathrm{p}=0.01)$, $\mathrm{RR}(\mathrm{p}=0.008), \mathrm{HR}(\mathrm{p}=0.04)$ y T $(\mathrm{p}=0.03)$. En cuanto a la PA, tanto la sistólica $(\mathrm{p}=0,47)$ como la diastólica $(\mathrm{p}=$ $0,46)$ no presentaron diferencias significativas. La PC proporcionó un aumento significativo de la SpO2 y una disminución significativa de la RR, la FC y la T en los recién nacidos prematuros evaluados.

Palabras clave: Prematuridad; Recién nacido; Posición canguro; Signos vitales.

\section{Introdução}

O termo pré-termo ou prematuridade é definido pela organização Mundial da Saúde (OMS) como aquele que ocorre antes de 37 semanas de gestação ao nascer, podendo ser classificada como extrema quando a gestação é inferior a 28 semanas; acentuada quando ocorre entre 28 e 31 semanas de gestação e moderada quando ocorre entre 32 e 36 semanas de idade gestacional (Almeida et al, 2013).

Segundo dados do Sistema Nacional de Nascidos Vivos Brasileiro, em 2011, cerca de 11,8\% de todos os nascimentos foram prematuros. A OMS contabiliza anualmente aproximadamente 280 mil nascimentos pré-termo no Brasil, tendo sua prevalência nos estados de Minas Gerais, São Paulo, Rio de Janeiro, Rio Grande do Sul e no Distrito Federal (Victora et al, 2013).

Cientificamente, os RNPT são fisiológica e metabolicamente imaturos e, portanto, vulneráveis, assim aumentando significativamente a taxa de mortalidade e morbidade em 4,6 vezes em relação aos recém-nascidos a termo, nascidos entre 37 e 42 semanas de gestação (Paulos \& Winckler, 2015).

Os recém-nascidos pré-termo necessitam, logo após o nascimento, cuidados hospitalares intensivos pela maior incidência de dificuldades alimentar, respiratória e cardíaca, icterícia, hipoglicemia, hipotermia, apneia, problemas imunológicos e também da realização de exames analíticos, necessitando com maior frequência de administração de fluidos endovenosos e necessidade de ventilação mecânica (Rodrigues \& Bolsoni-silva, 2011).

O desenvolvimento de Unidades de Terapia Intensiva (UTIs) para cuidados neonatais aumentam a taxa de sobrevida de prematuros, entretanto, mesmo com o avanço de tecnologias voltadas para essa área médica, os efeitos da prematuridade ao longo da visão são pouco conhecidas. O emprego de abordagens multidisciplinares e multiprofissionais o mais precoce possível se faz necessário e útil, objetivando amenizar e/ou prevenir sequelas, reduzindo o tempo de internação possibilitando a alta precoce das UTIs e das alas hospitalares (Coelli et al, 2011).

O Método Mãe Canguru, também conhecido como Cuidado Mãe canguru, Posição Canguru ou Contato Pele a Pele é um método voltado á assistência neonatal em recém-nascidos prematuros que consiste na colocação do bebê em contato direto pele a pele com a mãe. O Método foi idealizado e implantado pelos médicos Edgar Rey Sanabria e Hector Matinez em 1979 no Instituto Materno-Infantil de Bogotá e assim denominado devido à maneira pela qual as mães carregavam seus bebês após o nascimento, de forma semelhante aos marsupiais (Maia et al, 2011). No Brasil, o Método foi implantado em 1991 no Hospital Guilherme Álvaro em Santos, São Paulo e em seguida no Instituto Materno-Infantil de Pernambuco, no Recife e desde então, utilizado em vários serviços em todo o país. No mês de dezembro de 1999 o Método Canguru tem seu lançamento normatizado na Atenção Humanizada ao Recém-Nascidos de baixo Peso através da Portaria Ministerial no 693 de 05/07/2000 passando a ser inclusa na Política Governamental de Saúde Pública do Brasil como um procedimento de assistência médica (Arrivabene \& Tyrrell, 2010).

Durante a Posição Canguru, onde o recém-nascido é posicionado contra o peito da mãe, os principais objetivos são aumentar o vínculo mãe-filho; promover melhor controle térmico; diminuir o tempo de separação mãe-filho evitando longos períodos sem estimulação sensorial; estimular o aleitamento materno favorecendo maior frequência, precocidade e duração; corrigir as situações de risco como ganho inadequado de peso, sinais de refluxo, infecções e apneias; reduzir o estresse e a dor; diminuir o risco de infecções hospitalares permitindo assim alta precoce otimizando os leitos de UTI devido à maior 
rotatividade de leitos e consequentemente melhor qualidade da assistência com menor custo para o sistema de saúde (Boundy et al, 2016).

Mediante tais considerações, o presente estudo teve como objetivo geral, avaliar os efeitos da Posição Canguru sobre os sinais vitais no pré e pós a aplicação do método em recém-nascidos internados em um grande hospital da cidade de CuritibaPR.

\section{Metodologia}

Esta pesquisa possui natureza experimental, descritiva, comparativa e quantitativa. Foi realizada nas dependências da Unidade de Terapia Intensiva Neonatal de um grande Hospital localizado na Cidade de Curitiba-PR, após estar de acordo com a Resolução 196 no que diz respeito aos critérios éticos, sendo aprovado pelo CEP sob parecer consubstanciado número 2.133.511 e CAEE número 68724317.3.0000.5529, na data de 22/06/2017.

A população total participante deste estudo foi de 12 (doze) indivíduos, onde foram selecionados apenas os indivíduos que cumpriram os critérios de inclusão e exclusão. Foram incluídos neste estudo recém-nascidos, de ambos os sexos, com idade gestacional entre 29 e 37 semanas ao nascer, pesando entre 1000 e 2000 gramas, hemodinamicamente estáveis, que não possuíssem disfunções respiratórias, cardíacas e/ou neurológicas, que não foram submetidos a algum tipo de procedimento cirúrgico, que não fizessem uso de Ventilação Mecânica Não-Invasiva e que o responsável assinasse o Termo de Consentimento Livre e Esclarecido concordando em participar da pesquisa. Foram excluídos os indivíduos que não obedeceram a um ou mais dos itens citados anteriormente.

Após o responsável assinar o Termo de Consentimento Livre e Esclarecido concordando com a metodologia proposta pelos pesquisaᄀdores, os indivíduos foram avaliados por uma ficha de avaliação previamente elaborada. A ficha de avaliação foi composta dos seguintes itens: Identificação dos indivíduos, dados do recém-nascido e dados hemodinâmicos com as variáveis Temperatura, Saturação periférica de oxigênio, pressão arterial e frequências cardíaca e respiratória.

Para avaliar a saturação periférica de oxigênio $(\mathrm{SpO} 2)$ e a pressão arterial média, foi utilizado um monitor não invasivo configurável portátil multiparamétrico MINDRAY MEC-1000, acoplado a um sensor de oxímetro de pulso Moriya M1000 e a um manguito para avaliação da pressão arterial MINDRAY C1500. A temperatura corporal foi aferida por meio de um termômetro clínico G-TECH TH400 digital, graduado a cada $0.1^{\circ} \mathrm{C}$. A frequência Respiratória foi verificada com o uso de um cronômetro bem como o tempo de aplicação da Posição Canguru.

Para avaliação dos sinais vitais o recém-nascido foi mantido em incubadora com temperatura de $27^{\circ} \mathrm{C}$, em decúbito dorsal, utilizando sobre fralda, durante 30 minutos. Após atingir estado 3 (calmo) segundo a escala Prechtl (Brasil, 2016) que corresponde a olhos abertos, sem movimentos grosseiros, a coleta de dados foi realizada.

A temperatura corporal foi avaliada através do posicionamento do termômetro na prega axilar do recém-nascido, onde aguardou-se a emissão sonora do mesmo para a conclusão do teste.

Posicionando um sensor na face plantar do pé direito e um manguito entorno do braço esquerdo, para padronização, verificou-se a saturação periférica de oxigênio, a pressão arterial e frequência cardíaca. A verificação da frequência respiratória foi realizada durante um minuto cronometrado.

O recém-nascido foi posicionado verticalmente, entre as mamas e de frente para a mãe, com a cabeça lateralizada, os membros superiores flexionados, aduzidos, com cotovelos próximos ao tronco e membros inferiores flexionados e aduzidos. A mãe foi orientada a permanecer sentada com elevação da cabeça à aproximadamente 80 graus em poltrona hospitalar acolchoada. $\mathrm{O}$ recém-nascido permaneceu na posição canguru durante trinta minutos, sendo em seguida realizada nova coleta dos sinais vitais avaliados pré-procedimento.

Os dados obtidos foram analisados através do Teste $\mathrm{t}$ de Student, traduzindo em média \pm desvio padrão. $\mathrm{O}$ nível de 
significância adotado foi de $p<0,05$.

\section{Resultados e Discussão}

Foram avaliados 12 RNPT, sendo 6 do sexo feminino e 6 do sexo masculino. A média de peso ao nascer foi de $1,43 \pm 0,35$ quilogramas no grupo feminino e 1,75 $\pm 0,11$ entre o masculino, já a média da idade gestacional foi de $30,3 \pm 0,81$ semanas do grupo feminino e 30,6 $\pm 1,5$ no grupo masculino (Tabela 1 ).

Tabela 1 - Sexo, peso ao nascer e idade gestacional dos RNPT avaliados

\begin{tabular}{cccccccccc}
\hline & & \multicolumn{4}{c}{ Peso } & \multicolumn{4}{c}{ Idade Gestacional } \\
Sexo & N & Mínimo & Máximo & Média & Desvio-Padrão & Mínimo & Máximo & Média & Desvio-Padrão \\
\hline F & 6 & 1,03 & 1,85 & 1,43 & 0,35 & 29 & 31 & 30,3 & 0,81 \\
M & 6 & 1,57 & 1,9 & 1,75 & 0,11 & 29 & 31 & 30,6 & 1,5 \\
\hline
\end{tabular}

Fonte: Autores (2021).

A Tabela 2 demonstra aumento significativo da saturação periférica de oxigênio onde a média pré-procedimento era de $95,2 \%$ e após 30 minutos foi de $97 \%$ ( $\mathrm{p}=0,016)$ e diminuição significava das frequências respiratória e cardíaca e da temperatura, sendo $\mathrm{p}=0,008, \mathrm{p}=0,041$ e $\mathrm{p}=0,038$ respectivamente.

Já a variável pressão arterial não apresentou diferença estatisticamente significativa entre o pré e o pós-procedimento tanto na pressão arterial sistólica como na diastólica, onde observou-se p=0,473 na sistólica e p=0,461 na diastólica (Tabela 2).

Tabela 2 - Média dos sinais vitais e da oximetria

\begin{tabular}{cccc}
\hline Variáveis & PRÉ & PÓs & $\boldsymbol{P}$ \\
\hline SpO2 & $95,2 \pm 1,6$ & $97 \pm 1,5$ & $0,016^{*}$ \\
FR & $58,8 \pm 6,3$ & $52,2 \pm 9,4$ & $0,008^{*}$ \\
FC & $150,7 \pm 15,7$ & $142,7 \pm 11,9$ & $0,041^{*}$ \\
T & $36,3 \pm 0,2$ & $36,1 \pm 0,3$ & $0,038^{*}$ \\
PAS & $63,7 \pm 7,7$ & $63,4 \pm 11,4$ & 0,473 \\
PAD & $32,2 \pm 6$ & $32 \pm 6,5$ & 0,461 \\
\hline
\end{tabular}

Dados apresentados em média \pm Desvio Padrão. PRÉ: pré-procedimento; PÓS pós-procedimento; SpO2: Saturação Periférica de Oxigênio; FR: Frequência Respiratória; FC: Frequência Cardíaca; T: Temperatura corporal; PAS: Pressão Arterial Sistólica; PAD: Pressão Arterial Diastólica; $P$. Nivel de significância $(p<0,05) ;{ }^{*}$ valores estatisticamente significativos.

Fonte: Autores (2021).

A Posição Canguru mostra-se benéfica, quando comparada à técnicas convencionais utilizadas em RNPT. Seus benefícios envolvem a diminuição do risco de sepse, dor, mortalidade, hipotermia, hipoglicemia e readmissão hospitalar e ao aumento da probabilidade de amamentação precoce, ganho de peso e crescimento adequado da circunferência cefálica, além de proporcionar maior estabilização dos sinais vitais devido ao contato pele a pele (Vieira, 2017) 
Devido à imaturidade dos órgãos e sistemas dos RNPT na manutenção fisiológica, estes apresentam-se propensos a diversas complicações e predispostos à patologias oportunistas, assim, faz-se necessário a utilização de técnicas externas (Boju et al, 2012).

No presente estudo pode-se observar aumento significativo da $\mathrm{SpO}$, concordando com os estudos de Tenório et al, (2010), com 24 RNPT e Boju et al, (2012), com 86 RNPT. Este achado é explicado por Olmedo et al, (2012), que descreve o aumento da oxigenação tecidual durante a Posição Canguru pelo estado de relaxamento do bebê que provavelmente diminui o consumo de oxigênio. Padilha et al, (2014), ainda descrevem que os benefícios da Posição Canguru sobre a SpO2 estão diretamente associados à posição confortável e ao estado calmo que ocorre durante a aplicação do método pelo contato entre a mãe e bebê. Boundy et al, (2016), realizaram uma meta-análise onde observaram um aumento de 0,9\% na SpO2 em relação aos valores encontrados em grupos controle, associando este aumento com a diminuição da frequência respiratória.

Quando se avalia a variável FR, estudos apontam diminuição desta após a aplicação da Posição Canguru, mesmo resultado encontrado no presente estudo. Os mesmos autores explicam que a diminuição da FR ocorre pela estabilidade da caixa torácica obtida com o posicionamento adequado quando em contato pele a pele com a mãe, alterando também as funções hemodinâmicas do recém-nascido (Carvalho \& Zangão, 2014). O posicionamento estudado também favorece o sono, principalmente o profundo onde $52,3 \%$ dos recém-nascidos permanecem nesse estado, comparando aos períodos antes $(6,8 \%)$ e após $(13,6 \%)$. Olmedo et al, (2012). ainda descrevem que este achado se deva a pesar das condições anatômicas e fisiológicas dos recém-nascidos, comum nesta faixa etária, a qual predispõe a ocorrência de respirações periódicas, crises de apneia e distorções da caixa torácica.

Em relação a FC, a literatura não apresenta um consenso. Boju et al, (2012) e Olmedo et al, (2012), observaram diminuição significativa, Tenório et al, (2010), observaram diminuição, porém não significativa, mostrando estabilização, já para Boundy et al, (2016), a posição canguru não exerce efeito significativo sobre a FC média demonstrando uma diferença de 0,41 batimentos por minuto em grupo de 15 recém-nascidos estudos. Almeida et al, (2013), e Olmedo et al, (2012), observaram uma tendência à diminuição da FC, devido a ausência de estresse físico e psicológico do bebê enquanto este permanece junto ao seio materno durante a Posição Canguru. Como já citado, a FC está diretamente relacionada à FR, considerando que o bebê se encontra calmo e em sono tranquilo, porém, sugere também que estas variáveis são dados incertos, já que se sobrepõem aos padrões respiratórios e cardíacos da mãe.

Assim como a SpO2, FR e FC, a Temperatura (T) também exerce um papel fundamental para garantir um equilíbrio homeostático do RNPT. Na pesquisa realizada por Olmedo et al, (2012), os autores não observaram diferença significativa da T comparando o pré e pós técnica, já Carvalho e Zangão (2014) descrevem, em seu estudo, diminuição significativa $(\mathrm{p}<0,01)$ da T em 33 RNPT após 30 minutos da realização da Posição Canguru assim como o estudo de Boundy et al, (2016), que observou 78\% menos risco de hipotermia e $23 \%$ menos risco de hipertermia após a realização da Posição Canguru. Rolim et al, (2010), explicam que RNPT quando expostos à hipotermia aguda os mesmos respondem com vasoconstrição periférica causando metabolismo anaeróbio e acidose metabólica podendo produzir constrição dos vasos pulmonares resultando em hipóxia, metabolismo anaeróbico e acidose adicionais. Casati et al, (2010), ainda explicam que os RNPT possuem dificuldade na manutenção da temperatura corporal, em virtude, principalmente, da falta de sudorese, da produção defeituosa de calor por menor movimentação, da imaturidade dos centros nervosos, da escassez de tecido celular subcutâneo e da oferta de oxigênio, limitada por distúrbios respiratórios. A Posição Canguru evita a perda de calor corporal e está associada com a manutenção ou aumento do calor, onde o contato íntimo do bebê com o corpo da mãe ajuda na regulação da temperatura corporal da criança (Casati et al, 2010). Diante dos achados e das explicações acredita-se que a estabilização da temperatura se deve ao fato que a Posição Canguru evita a perda de calor corporal excessivo, favorecendo a homeostasia que é regulada pelo balanço entre a termogênese e a termólise, além de estar associada à manutenção ou aumento de calor durante a técnica (Azevedo et al, 2011). 
Em relação à Pressão Arterial (PA) tanto sistólica (PAS) como a Diastólica (PAD) não observamos diferença estatística entre o pré e pós procedimento, mesmo resultado encontrado nos estudos de Almeida et al, (2013) e Gazzoto et al, (2000), já Tenório et al, (2010), observaram diminuição significativa $(\mathrm{p}=0,01)$ da PA onde explicam que a mesma é influenciada por vários fatores como peso ao nascer, idade gestacional, idade pós-natal e gestação múltipla. Explica-se a dificuldade das medidas e interpretação da PA em crianças, especialmente em neonatos, o tamanho do braço, o qual requer a avaliação e seleção de um manguito de tamanho apropriado e leituras de difícil interpretação, espacialmente em recémnascidos chorosos dando importância a utilização no nível 3 da escala comportamental de Prechtl. Outro procedimento é realizar uma única medida da PA média afim de tornar mais fácil a sua interpretação em relação à mudanças nas pressões sistólica e diastólica, as quais muitas vezes movem-se em diferentes direções, onde a mesma tende a empurrar o sangue através da circulação sistêmica (Ribeiro et al, 2007).

\section{Conclusão}

Com base nos dados oriundos da pesquisa, se chegou à conclusão que após a aplicação da Posição Canguru em RNPT, o mesmo promoveu aumento significativo da SpO2, e diminuição significativa da FR, FC e da T enquanto a variável PA não sofreu alteração significativa, podendo esta, estar relacionada à dificuldade de aferição, pouco tempo observação e/ou número da amostra.

Salvo as limitações da pesquisa, a Posição Canguru mostra-se eficaz, podendo ser aplicada em qualquer hospital, por seu baixo custo, trazendo grandes benefícios para a manutenção e recuperação do RNPT.

Desse modo, os dados coletados no ano de 2017, são atuais e relevantes para o ano vigente. Visto que o método empregado nos recém-nascidos pré-termo é eficaz, trazendo inúmeros benefícios para o mesmo, com o desenvolvimento extrauterino. Com o passar do tempo as Instituições de saúde, continuam incentivando o método nas unidades de cuidados intensivos e intermediários, visando a reabilitação desse indivíduo.

\section{Referências}

Almeida, T. S. O, Camelo, A. L, Lins, R. P, \& Mello, D. C. C. L. (2013). Investigação sobre os fatores de risco da prematuridade: uma revisão sistêmica. $R$ bras ci Saude, 17(3):301-8. https://periodicos.ufpb.br/ojs/index.php/rbcs/article/view/13674

Arrivabene, J. C., \& Tyrrell, M. A. R. (2010). Método Mãe Canguru: vivências maternas e contribuições para a enfermagem. Rev Latino-Am Enf; 18(2):13036. https://www.scielo.br/j/rlae/a/sBZRV7KZF4dtnZpMjw9jB8v/?format=pdf\&lang=pt

Azevedo, V. M. G. O., David, R. B., \& Xavier, C. C. (2011). Cuidado mãe-canguru em recém-nascidos pré-termo sob suporte ventilatório: avaliação dos estados comportamentais. Rev Bras Saúde Matern Infant (Recife);1(2):133-8. https://www.scielo.br/j/rbsmi/a/zW7VSWkqfHmQBY3HLGX6JZn/?lang=pt

Boju, S. L., et al (2012). Short spell kangaroo mother care and its differential physiological influence in subgroups of preterm babies. J Trop Pediatr. 2012;58(3):189-93. https://pubmed.ncbi.nlm.nih.gov/21908546/

Boundy, E. D., et al (2016). Kangoroo Mother care and Neonatal outcomes: A meta-analysis. Pediatrics 2016; 137(1):1-16. https://portaldeboaspraticas.iff.fiocruz.br/biblioteca/kangaroo-mother-care-and-neonatal-outcomes-a-meta-analysis/

Brasil (2016). Ministério da saúde. Secretaria de atenção à saúde. Diretrizes de estimulação precoce: crianças de zero e 3 anos com atraso do desenvolvimento neuropsicomotor decorrente da microcefalia. Brasília: Ministério da Saúde, 123.

https://bvsms.saude.gov.br/bvs/publicacoes/diretrizes_estimulacao_criancas_0a3anos_neuropsicomotor.pdf

Carvalho, A. M. V. G. O., \& Zangão, M. O. B. (2014). Contributo do contacto pele-a-pele na temperatura do recém-nascido. Rev Assoc Portug Enf Obst;14:63-8. https://dspace.uevora.pt/rdpc/bitstream/10174/13591/1/APEO-2014-Contributo-do-contacto-pele-a-pele-na-temperatura.pdf

Casati, P. S., Oliveira, C. S., \& Paula, S. (2010). Método mãe canguru e suas associações nos benefícios dos recém-nascidos de baixo peso. UNICiências; 14(1):135-46. file:///C:/Users/DELL/Downloads/3477-18249-1-PB\%20(1).pdf

Coelli, A. P., Nascimento, L. R., Mill, J. G., \& Molina, M. C. B. (2011). Prematuridade como fator de risco para pressão arterial elevada em crianças: uma revisão sistemática. Cad Saúde Pública. 27(2): 207-18. https://www.scielo.br/j/csp/a/wCmYt9gRsbhb5ZNT3pnprvN/?lang=pt\&format=pdf

Gazzoto, D., Masetti, P., \& Meli, M. (2000). Kangaroo care improves post-extubation cardiorespiratory parameters in infants after open-heart surgery. Acta Pediatr: 89:728-9. https://pubmed.ncbi.nlm.nih.gov/10914972/ 
Research, Society and Development, v. 11, n. 2, e9211225387, 2022

(CC BY 4.0) | ISSN 2525-3409 | DOI: http://dx.doi.org/10.33448/rsd-v11i2.25387

Maia, J. A., Oliveira, M. P., Furtado, S. S., Silva, L. M., \& Pereira, M. L. B. (2011). Método Canguru: a importância da família na recuperação do recémnascido de baixo peso. Enfermagem em Foco; 2(4):231-34. http://revista.cofen.gov.br/index.php/enfermagem/article/view/190

Olmedo, M. D., Gabas, G. S, Merey, L. S. F., et al (2012). Respostas fisiológicas de recém-nascidos pré-termo submetidos ao Método Mãe-Canguru e posição prona. Fisioter Pesq. 2012;19(2):115-21. https://periodicos.unifor.br/RBPS/article/view/2731

Padilha, J. F., Steidl, S. E. M., \& Braz, M. M. (2014). Efeitos do método mãe-canguru em recém-nascidos pré-termo. Fisioter Brasil, 15(2):147-52. file://C:/Users/DELL/Downloads/330-Texto\%20do\%20Artigo-1570-1-10-20160706.pdf

Paulos, L. M. P, \& Winckler, L. (2015). Prematuridade tardia: estarão eles preparados para nascer? Res Ped. 5(1):10-3. http://www.residenciapediatrica.com.br/detalhes/134/prematuridade-tardia--estarao-eles-preparados-para-nascer-

Ribeiro, M. A. S., Garcia, P. C. R., \& Fiori, R. M (2007). Determinação de pressão arterial em recém-nascidos. Sci Med;17(3):156-67. file:///C:/Users/DELL/Downloads/1665-Article\%20Text-9986-1-10-20080327.pdf

Rodrigues, O. M. P. R., \& Bolsoni-silva, A.T. (2011). Efeitos da prematuridade sobre o desenvolvimento de lactentes. Rev Bras crescimento desenvolv hum. São Paulo, 21(1):111-21. http://pepsic.bvsalud.org/scielo.php?script=sci_arttext\&pid=S0104-12822011000100011

Rolim, K. M. C., et al (2010). Cuidado quanto à termorregulação do recém-nascidos prematuro: o olhar da Enfermagem. Rev Rene Fortaleza 2010;11(2):4452. http://www.periodicos.ufc.br/rene/article/view/4521

Tenório, E. A. M., et al (2010). Avaliação dos parâmetros fisiológicos em recém-nascidos pré-termos de baixo peso antes e após a aplicação do método mãecanguru. Rev Fisioter Brasil. 2010: 11(1):44-47. file:///C:/Users/DELL/Downloads/1331-Texto\%20do\%20Artigo-8085-1-10-20171114.pdf

Victora, C., Barros, F., Matijasevich, A., Silveira, M. et al, (2013). Consultoria: Pesquisa para estimar a prevalência de nascimentos pré-termo do Brasil e explorar possíveis causas. Programa de Pós-Graduação em Epidemiologia. Universidade Federal de Pelotas, Pelotas-RS, p.18. http://scielo.iec.gov.br/pdf/ess/v22n4/v22n4a02.pdf

Vieira, T. P. (2017). Participação da família na aplicação do método canguru: revisão integrativa. Universidade Federal do Maranhão p. 49. https://rosario.ufma.br/jspui/bitstream/123456789/1302/1/TaissaVieira.pdf 